Georgian Mathematical Journal

1(1994), No. 4, 353-365

\title{
OPTIMAL TRANSMISSION OF GAUSSIAN SIGNALS THROUGH A FEEDBACK CHANNEL
}

\author{
O. GLONTI
}

\begin{abstract}
Using the methodology and results of the theory of filtering of conditionally Gaussian processes, the optimal schemes of transmission of Gaussian signals through the noisy feedback channel are constructed under the new power conditions.
\end{abstract}

In the present paper, in contrast to our previous work [1], the problem of transmission of Gaussian signals through the noisy feedback channel under new power conditions (see conditions (15),(38) and (40)) is investigated. The obtained results, in our opinion, imply significant simplification and better clarity.

In $\S 1$ the optimal (in the sense of mean square criterion) linear schemes of transmission in the case of discrete time are constructed. In $\S 2$ the optimal linear schemes of transmission in the case of continuous time are investigated and it is proved that these schemes are also optimal in the general class of transmission schemes.

\section{$\S 1$. Optimal Transmission in the Discrete Case}

1. Let the signal $\theta=\left(\theta_{t}, \mathcal{F}_{t}\right), t \in S=\{0, \Delta, 2 \Delta, \ldots, T\}, \Delta>0$, be a discrete Gaussian process given on the basic probability space $(\Omega, \mathcal{F}, P)$ with the nondecreasing family of $\sigma$-algebras $\left(\mathcal{F}_{t}\right), t \in S, \mathcal{F}_{t} \subseteq \mathcal{F}, t<s$, satisfying the following finite difference equation:

$$
\Delta \theta_{t}=a(t) \theta_{t} \Delta+b(t) \Delta v_{t},
$$

where $v=\left(v_{t}, \mathcal{F}_{t}\right), t \in S$, is a Gaussian random sequence (G.R.S.) $N(0, t)$ with independent increments independent of $\theta_{0}$, which is a Gaussian $N(m, \gamma)$, $\gamma>0$, random variable; $a(t)$ and $b(t)$ are known functions on $S$, for every $t$ $|a(t)| \leq k,|b(t)| \leq k$, where $k$ is some constant.

Suppose that $\theta$ is transmitted according to the scheme

$$
\Delta \xi_{t}=\left[A_{0}(t, \xi)+A_{1}(t, \xi) \theta_{t}\right] \Delta+\Delta w_{t}, \quad \xi_{0}=0,
$$

1991 Mathematics Subject Classification. 60G35,93E20,94A40. 
where $w=\left(w_{t}, \mathcal{F}_{t}\right), t \in S$, is a G.R.S. $N(0, t)$ with independent increments which is independent of $\theta_{0}$ and $v$. The nonanticipating (with respect to $\xi$ ) functionals $A_{0}(t, \xi)$ and $A_{1}(t, \xi)$ define the coding.

The transmission performed according to the scheme (2) is a transmission of a Gaussian message $\theta$ through a noiseless feedback channel which is an analogue of the additive "White noise" channel in the discrete time case. No instantaneous feedback is required here (which is essential in the continuous time), but we assume that the quantization step $\Delta$ is equal to the time of signal return.

Suppose that the coding functionals $A_{0}$ and $A_{1}$ satisfy the condition

$$
E\left[A_{0}(t, \xi)+A_{1}(t, \xi) \theta_{t}\right]^{2} \leq p,
$$

where $p$ is a constant characterizing the energetic potential of the transmitter.

Consider the decoding $\hat{\theta}=\hat{\theta}_{t}(\xi)$ satisfying for every $t$ the condition

$$
E \hat{\theta}_{t}^{2}<\infty
$$

Such a kind of $\left[\left(A_{0}, A_{1}\right), \hat{\theta}\right]$ forms a class of admissible codings and decodings.

The problem is to find the codings $\left(A_{0}^{*}, A_{1}^{*}\right)$ and the decodings $\hat{\theta}^{*}$ optimal in the sense of the criterion

$$
\delta(t)=\inf _{A_{0}, A_{1}, \hat{\theta}} E\left[\theta_{t}-\hat{\theta}_{t}(\xi)\right]^{2}
$$

where inf is taken in the class of all admissible $\left[\left(A_{0}, A_{1}\right), \hat{\theta}\right]$.

Theorem 1. During the transmission a discrete Gaussian process described by the finite difference equation (1) according to the transmission scheme (2) under conditions (3),(4) and the optimal coding functionals $A_{0}^{*}$ and $A_{1}^{*}$, have the form

$$
A_{1}^{*}(t)=\sqrt{\frac{p}{\gamma}}(1+p \Delta)^{\frac{t}{2 \Delta}}, \quad A_{0}^{*}\left(t, \xi^{*}\right)=-A_{1}^{*}(t) m_{t}^{*},
$$

where the optimal decoding $\hat{\theta}_{t}^{*}=m_{t}^{*}=E\left[\theta_{t} \mid \mathcal{F}_{t}^{\xi^{*}}\right]$ and the transmitted signal are defined by the relations

$$
\begin{aligned}
\Delta m_{t}^{*} & =a(t) m_{t}^{*} \Delta+\sqrt{p \gamma_{t}^{*}} \frac{(1+a(t) \Delta)}{1+p \Delta} \Delta \xi_{t}^{*}, \quad m_{0}^{*}=m, \\
\Delta \xi_{t}^{*} & =\sqrt{\frac{p}{\gamma_{t}^{*}}}\left(\theta_{t}-m_{t}^{*}\right) \Delta+\Delta w_{t}, \quad \xi_{0}^{*}=0 .
\end{aligned}
$$


The minimal error of message reproduction has the following form:

$$
\begin{gathered}
\delta(t)=\gamma_{t}^{*}=\gamma\left(\prod_{k=0}^{t-\Delta}(1+a(k) \Delta)^{2}\right)(1+p \Delta)^{-\frac{t}{\Delta}}+ \\
+\sum_{k=0}^{t-\Delta}\left(b^{2}(k) \Delta\right)\left(\prod_{s=k+\Delta}^{t-\Delta}(1+a(s) \Delta)^{2}\right)(1+p \Delta)^{-\frac{t-k-2 \Delta}{\Delta}} .
\end{gathered}
$$

Proof. For the given $A_{0}$ and $A_{1}$ it is known that $\hat{\theta}_{t}=m_{t}=E\left(\theta_{t} \mid \mathcal{F}_{t}^{\xi}\right)$. Hence $\delta(t)=\inf _{\left(A_{0}, A_{1}\right)} E\left(\theta_{t}-m_{t}\right)^{2}=\inf _{\left(A_{0}, A_{1}\right)} E \gamma_{t}$.

In order to find $m_{t}$ and $\gamma_{t}=E\left[\left(\theta_{t}-m_{t}\right)^{2} \mid \mathcal{F}_{t}^{\xi}\right]$ we shall use filtering equations for the conditionally Gaussian random sequence (see [1] or [2]).

The rest of the proof is similar to that of the theorem on the optimal scheme for the transmission of Gaussian processes through a noiseless feedback channel in continuous time (see [2]).

2. It is natural to investigate the case of Gaussian signal transmission when white noise is imposed on the back signal, i.e., the message is transmitted according to the scheme

$$
\Delta \xi_{t}=A(t, \theta, \widetilde{\xi}) \Delta+\sigma(t) \Delta w_{t}, \quad \xi_{0}=0,
$$

where the back signal $\widetilde{\xi}_{t}$ has the form, say, $\widetilde{\xi}_{t}=\xi_{t}+\eta_{t}$ or $\widetilde{\xi}_{t}=\Pi(t, \xi)+\eta_{t}$, where $\Pi(t, \xi)$ is some nonanticipating functional and $\eta_{t}$ is the noise in the back channel.

We shall specify the problem under consideration.

Let the signal $\theta=\left(\theta_{t}, \mathcal{F}_{t}\right), t \in S$, be a discrete Gaussian process described by the equation (1).

Assume that $\theta$ is transmitted according to the scheme

$$
\Delta \xi_{t}=\left[A_{0}(t) \widetilde{\xi}_{t}+A_{1}(t) \theta_{t}\right] \Delta+\sigma(t) \Delta w_{t}, \quad \xi_{0}=0,
$$

where the functions $A_{0}$ and $A_{1}$ define the coding.

The back signal has the form

$$
\widetilde{\xi}_{t}=\Pi(t, \xi)+\eta_{t}
$$

Here $\Pi(t, \xi)$ is a transformator of the back message and $\eta_{t}$ is the noise in the back channel governed by the finite difference equation

$$
\Delta \eta_{t}=c(t) \eta_{t} \Delta+d(t) \Delta \bar{w}_{t},
$$

where $\bar{w}=\left(\bar{w}_{t}, \mathcal{F}_{t}\right), t \in S$, is a G.R.S. $N(0, t)$ with independent increments, for every $t|c(t)| \leq L$ and $|d(t)| \leq L$, where $L$ is some constant. 
Denote

$$
\begin{gathered}
m_{t}^{(1)}=m_{t}=E\left[\theta_{t} \mid \mathcal{F}_{t}^{\xi}\right], \quad m_{t}^{(2)}=E\left[\eta_{t} \mid \mathcal{F}_{t}^{\xi}\right], \\
\gamma_{t}^{(1)}=\gamma_{t}=E\left[\left(\theta_{t}-m_{t}\right)^{2} \mid \mathcal{F}_{t}^{\xi}\right], \quad \gamma_{t}^{(2)}=E\left[\left(\eta_{t}-m_{t}^{(2)}\right)^{2} \mid \mathcal{F}_{t}^{\xi}\right], \\
\gamma_{t}^{(1,2)}=\gamma_{t}^{(2,1)}=E\left[\left(\theta_{t}-m_{t}\right)\left(\eta_{t}-m_{t}^{(2)}\right) \mid \mathcal{F}_{t}^{\xi}\right], \\
\widetilde{\gamma}_{t}=\gamma_{t}^{(2)}-\frac{\gamma_{t}^{2}(1,2)}{\gamma_{t}}, \quad \hat{\gamma}_{t}=\frac{\gamma_{t}^{(1,2)}}{\gamma_{t}} .
\end{gathered}
$$

The problem is to find the codings $\left(A_{0}^{*}, A_{1}^{*}\right)$, decodings $\hat{\theta}^{*}=\left(\hat{\theta}_{t}^{*}\left(\xi^{*}\right)\right)$, $t \in S$, and the transformator $\Pi^{*}$ optimal in the sense of the square criterion

$$
\delta(t)=\inf _{A_{0}, A_{1}, \Pi, \hat{\theta}} E\left[\theta_{t}-\hat{\theta}_{t}(\xi)\right]^{2},
$$

where inf is taken in the class of admissible $A_{0}, A_{1}, \Pi, \hat{\theta}$ for which the power condition $^{1}$

$$
\begin{gathered}
E\left[A_{0}(t) \widetilde{\xi}_{t}+A_{1}(t) \theta_{t}\right]^{2} \leq p(t) \\
A_{0}^{2}(t) \widetilde{\gamma}_{t} \geq q(t)
\end{gathered}
$$

holds where $p(t)$ and $q(t)$ are summable functions on $S$ characterizing the changes of the energetic transmitter potential, and $q(t) \leq p(t), t \in S$. Let

$$
E \hat{\theta}_{t}^{2}<\infty
$$

Theorem 2. When the Gaussian random sequence $\theta=\left(\theta_{t}, \mathcal{F}_{t}\right), t \in S$, governed by the finite difference equation (1) is transmitted according to the scheme of transmission (10)-(12) through a noisy feedback channel under conditions (14)-(16), then the optimal [in the sense of square criterion (13)] coding functions $A_{0}^{*}, A_{1}^{*}$, decoding functional, $\hat{\theta}^{*}$ and transformator of the back message $\Pi^{*}$ have the form

$$
\begin{gathered}
A_{0}^{*}(t)=\left(\frac{q(t)}{\widetilde{\gamma}_{t}^{*}}\right)^{\frac{1}{2}}, \quad A_{1}^{*}(t)=-\left(\frac{q(t)}{\widetilde{\gamma}_{t}^{*}}\right)^{\frac{1}{2}} \hat{\gamma}_{t}^{*}+\left(\frac{p(t)-q(t)}{\gamma_{t}^{*}}\right)^{\frac{1}{2}}, \\
\Pi^{*}(t, \xi)=-m_{t}^{*(2)}-\frac{A_{1}^{*}(t)}{A_{0}^{*}(t)} m_{t}^{*}, \quad \hat{\theta}_{t}^{*}\left(\xi^{*}\right)=m_{t}^{*}, \\
\Delta m_{t}^{*}=a(t) m_{t}^{*} \Delta+(1+a(t) \Delta)\left[\gamma_{t}^{*}(p(t)-q(t))\right]^{\frac{1}{2}} \times \\
\times\left(\sigma^{2}(t)+p(t) \Delta\right)^{-1} \Delta \xi_{t}^{*}, \quad m_{0}^{*}=m .
\end{gathered}
$$

${ }^{1}$ The fact that condition (15) is the power condition indeed will be shown at the end of the section. 
The optimal transmission has the following form:

$$
\begin{gathered}
\Delta \xi_{t}^{*}=\left\{\left(\frac{q(t)}{\widetilde{\gamma}_{t}^{*}}\right)^{\frac{1}{2}}\left(\eta_{t}-m_{t}^{*(2)}\right)+\left[-\left(\frac{q(t)}{\widetilde{\gamma}_{t}^{*}}\right)^{\frac{1}{2}} \hat{\gamma}_{t}^{*}+\left(\frac{p(t)-q(t)}{\gamma_{t}^{*}}\right)^{\frac{1}{2}}\right] \times\right. \\
\left.\times\left(\theta_{t}-m_{t}^{*}\right)\right\} \Delta+\sigma(t) \Delta w_{t}, \quad \xi_{0}^{*}=0,
\end{gathered}
$$

where $m_{t}^{*(2)}$ satisfies the finite difference equation

$$
\begin{gathered}
\Delta m_{t}^{*(2)}=c(t) m_{t}^{*(2)} \Delta+(1+c(t) \Delta)\left\{\hat{\gamma}_{t}^{*}\left[\gamma_{t}^{*}(p(t)-q(t))\right]^{\frac{1}{2}}+\left[q(t) \hat{\gamma}_{t}^{*}\right]^{\frac{1}{2}}\right\} \times \\
\times\left(\sigma^{2}(t)+p(t) \Delta\right)^{-1} \Delta \xi_{t}^{*}, \quad m_{0}^{*(2)}=m^{(2)}=E \eta_{0}^{(2)}
\end{gathered}
$$

and $\widetilde{\gamma}_{t}^{*}=\gamma_{t}^{*(2)}-\frac{\left(\gamma_{t}^{*(1,2)}\right)^{2}}{\gamma_{t}^{*}}$ and $\hat{\gamma}_{t}^{*}=\frac{\gamma_{t}^{*(1,2)}}{\gamma_{t}^{*}}$ are defined by the filtering equations (19)-(23) given below, where $A_{0}^{*}$ and $A_{1}^{*}$ are substituted from (17).

The minimal message reproduction error $\delta(t)$ has the form

$$
\begin{aligned}
& \delta(t)=\gamma_{t}^{*}=\gamma \prod_{k=0}^{t-\Delta}(1+a(k) \Delta)^{2}\left(\sigma^{2}(k)+q(k) \Delta\right)\left(\sigma^{2}(k)+p(k) \Delta\right)^{-1}+ \\
& +\sum_{k=0}^{t-\Delta} b^{2}(k)\left(\prod_{m=k+\Delta}^{t-\Delta}(1+a(m) \Delta)^{2}\left(\sigma^{2}(m)+q(m) \Delta\right)\left(\sigma^{2}(m)+p(m) \Delta\right)^{-1}\right) .
\end{aligned}
$$

Corollary. Let $a(t)=b(t) \equiv 0$, i.e., according to the scheme (10)-(12), a Gaussian $N(m, \gamma), \gamma>0$, random variable $\theta$ is transmitted. Then the reproduction error $\delta(t)$ is

$$
\delta(t)=\gamma \prod_{k=0}^{t-\Delta}\left(1+\frac{q(k)}{\sigma^{2}(k)} \Delta\right)\left(1+\frac{p(k)}{\sigma^{2}(k)} \Delta\right)^{-1} .
$$

In this case $\widetilde{\gamma}_{t}^{*}$ and $\hat{\gamma}_{t}^{*}$ satisfy the following finite difference equations:

$$
\begin{gathered}
\frac{\Delta \widetilde{\gamma}_{t}^{*}}{\Delta}=d^{2}(t)+\widetilde{\gamma}_{t}^{*}\left(c(t) \sigma^{2}(t)-q(t)\right)\left(\sigma^{2}(t)+q(t) \Delta\right)^{-1}, \quad \widetilde{\gamma}_{0}^{*}=0, \\
\frac{\Delta \hat{\gamma}_{t}^{*}}{\Delta}=\left\{\hat{\gamma}_{t}^{*}\left[c(t) \sigma^{2}(t)-q(t) \gamma_{t}^{*}+q(t)(1+c(t) \Delta)\right]-\right. \\
\left.-(1+c(t) \Delta)\left(\frac{q(t)}{\gamma_{t}^{*}}\right)^{\frac{1}{2}}\left(\frac{p(t)-q(t)}{\gamma_{t}^{*}}\right)^{\frac{1}{2}} \widetilde{\gamma}_{t}^{*}\right\}\left[\sigma^{2}(t)+q(t) \Delta\right]^{-1}, \quad \hat{\gamma}_{0}^{*}=0 .
\end{gathered}
$$

Proof of the Theorem. It can be easily seen that

$$
\delta(t)=\inf _{\left(A_{0}, A_{1}, \Pi\right)} E \gamma_{t}=\inf _{\left(A_{0}, A_{1}, \Pi\right)} \gamma_{t} .
$$

Rewrite (10) in the following form:

$$
\Delta \xi_{t}=\left[A_{0}(t) \Pi(t, \xi)+A_{0}(t) \eta_{t}+A_{1}(t) \theta_{t}\right] \Delta+\sigma(t) \Delta w_{t}, \quad \xi_{0}=0 .
$$


Then one can see that the equation of optimal filtering of a partially observable conditionally Gaussian process $\left(\widetilde{\theta}_{t}, \xi_{t}\right), t \in S$, with an unobservable component $\widetilde{\theta}_{t}=\left(\theta_{t}, \eta_{t}\right)$ (see [1] or [2]) leads to the following closed system of finite difference equations:

$$
\begin{gathered}
\Delta m_{t}=a(t) m_{t} \Delta+(1+a(t) \Delta)\left(A_{1}(t) \gamma_{t}+A_{0}(t) \gamma_{t}^{(1,2)}\right) \times \\
\times\left[\sigma^{2}(t)+\left(A_{1}^{2}(t) \gamma_{t}+2 A_{1}(t) A_{0}(t) \gamma_{t}^{(1,2)}+A_{0}^{2}(t) \gamma_{t}^{(2)}\right) \Delta\right]^{-1} \times \\
\times\left[\Delta \xi_{t}-\left(A_{0}(t)\left(\Pi(t, \xi)+m_{t}^{(2)}\right)+A_{1}(t) m_{t}\right) \Delta\right], \quad m_{0}=m, \\
\Delta m_{t}^{(2)}=c(t) m_{t}^{(2)} \Delta+(1+c(t) \Delta)\left(A_{1}(t) \gamma_{t}^{(1,2)}+A_{0}(t) \gamma_{t}^{(2)}\right) \times \\
{\left[\sigma^{2}(t)+\left(A_{1}^{2}(t) \gamma_{t}+2 A_{1}(t) A_{0}(t) \gamma_{t}^{(1,2)}+A_{0}^{2}(t) \gamma_{t}^{(2)}\right) \Delta\right]^{-1} \times} \\
\times\left[\Delta \xi_{t}-\left(A_{0}(t)\left(\Pi(t, \xi)+m_{t}^{(2)}\right)+A_{1}(t) m_{t}\right) \Delta\right], \quad m^{(2)}=m, \\
\quad \frac{\Delta \gamma_{t}^{(1)}}{\Delta}=b^{2}(t)+a^{2}(t) \gamma_{t}^{(1)} \Delta+2 a(t) \gamma_{t}^{(1)}- \\
-(1+a(t) \Delta)^{2}\left(A_{1}(t) \gamma_{t}^{(1)}+A_{0}(t) \gamma_{t}^{(1,2)}\right)^{2}\left[\sigma^{2}(t)+\right. \\
\left.+\left(A_{1}^{2}(t) \gamma_{t}^{(1)}+2 A_{1}(t) A_{0}(t) \gamma_{t}^{(1,2)}+A_{0}^{2}(t) \gamma_{t}^{(2)}\right) \Delta\right]^{-1}, \\
\gamma_{0}^{(1)}=\gamma, \\
\quad \frac{\Delta \gamma_{t}^{(2)}=d^{2}(t)+c^{2}(t) \gamma_{t}^{(2)} \Delta+2 c(t) \gamma_{t}^{(2)}-}{\Delta}-(1+c(t) \Delta)\left(A_{1}(t) \gamma_{t}^{(1,2)}+A_{0}(t) \gamma_{t}^{(2)}\right)^{2}\left[\sigma^{2}(t)+\right. \\
\left.+\left(A_{1}^{2}(t) \gamma_{t}^{(1)}+2 A_{1}(t) A_{0}(t) \gamma_{t}^{(1,2)}+A_{0}^{2}(t) \gamma_{t}^{(2)}\right) \Delta\right]^{-1}, \\
\gamma_{0}^{(2)}=\gamma_{2}, \\
\frac{\gamma_{t}^{(1,2)}}{\Delta}=a(t) \gamma_{t}^{(1,2)}+c(t) \gamma_{t}^{(1,2)}-(1+a(t) \Delta)(1+c(t) \Delta) \times \\
\times\left(A_{1}(t) \gamma_{t}^{(1)}+A_{0}(t) \gamma_{t}^{(1,2)}\right)\left(A_{1}(t) \gamma_{t}^{(1,2)}+A_{0}(t) \gamma_{t}^{(2)}\right)\left[\sigma^{2}(t)+\right. \\
\left.\left.+A_{1}^{2}(t) \gamma_{t}^{(1)}+2 A_{1}(t) A_{0}(t) \gamma_{t}^{(1,2)}+A_{0}^{2}(t) \gamma_{t}^{(2)}\right) \Delta\right]^{-1} .
\end{gathered}
$$

Equation (21) can be reduced to the form

$$
\begin{gathered}
\gamma_{t}=\left(\prod _ { k = 0 } ^ { t - \Delta } ( 1 + a ( k ) \Delta ) ^ { 2 } ( \sigma ^ { 2 } ( k ) + A _ { 0 } ^ { 2 } ( k ) \widetilde { \gamma } _ { k } \Delta ) \left[\sigma^{2}(k)+\right.\right. \\
\left.\left.+\left(A_{1}^{2}(k) \gamma_{k}+2 A_{1}(k) A_{0}(k) \gamma_{k}^{(1,2)}+A_{0}^{2}(k) \gamma_{k}^{(2)}\right) \Delta\right]^{-1}\right) \times \\
\times\left[\sum _ { l = 0 } ^ { t - \Delta } b ^ { 2 } ( l ) \left(\prod_{m=0}^{l}(1+a(m) \Delta)^{2}\left(\sigma^{2}(m)+A_{0}^{2}(m) \widetilde{\gamma}_{m} \Delta\right) \times\right.\right.
\end{gathered}
$$




$$
\begin{aligned}
\times\left[\sigma^{2}(m)\right. & +\left(A_{1}^{2}(m) \gamma_{m}+2 A_{1}(m) A_{0}(m) \gamma_{m}^{(1,2)}+\right. \\
& \left.\left.\left.\left.+A_{0}^{2}(m) \gamma_{m}^{(2)}\right) \Delta\right]^{-1}\right)^{-1}+\gamma\right] .
\end{aligned}
$$

Using inequality (15) and the inequality resulting from (14)

$$
A_{1}^{2}(t) \gamma_{t}+2 A_{1}(t) A_{0}(t) \gamma_{t}^{(1,2)}+A_{0}^{2}(t) \gamma_{t}^{(2)} \leq p
$$

we obtain from (24) $E\left(\theta_{t}-m_{t}\right)^{2}=\gamma_{t} \geq \psi(t)$, where

$$
\begin{gathered}
\psi(t)=\left(\prod_{k=0}^{t-\Delta}(1+a(k) \Delta)^{2}\left(\sigma^{2}(k)+q(k) \Delta\right)\left(\sigma^{2}(k)+p(k) \Delta\right)^{-1}\right) \times \\
\times\left[\sum _ { l = 0 } ^ { t - \Delta } b ^ { 2 } ( l ) \left(\prod_{m=0}^{l}(1+a(m) \Delta)^{2}\left(\sigma^{2}(m)+q(m) \Delta\right) \times\right.\right. \\
\left.\left.\times\left(\sigma^{2}(m)+p(m) \Delta\right)^{-1}\right)^{-1}+\gamma\right]
\end{gathered}
$$

and since $\psi(t)$ is a known function, we have

$$
\delta(t) \geq \psi(t)
$$

for all $t \in S$.

The equality in (26) is obtained when

$$
\begin{gathered}
A_{0}(t) \widetilde{\gamma}_{t}=q(t), \\
A_{1}^{2}(t) \gamma_{t}+2 A_{1}(t) A_{0}(t) \gamma_{t}^{(1,2)}+A_{0}^{2}(t) \gamma_{t}^{(2)}=p(t) .
\end{gathered}
$$

Since

$$
\begin{gathered}
p(t) \geq E\left(A_{0}(t) \widetilde{\xi}_{t}+A_{1}(t) \theta_{t}\right)^{2}=E\left[A_{0}(t) \Pi(t, \xi)+\right. \\
\left.+A_{0}(t) m_{t}^{(2)}+A_{1}(t) m_{t}\right]^{2}+A_{1}^{2}(t) \gamma_{t}+2 A_{1}(t) A_{0}(t) \gamma_{t}^{(1,2)}+A_{0}^{2}(t) \gamma_{t}(2),
\end{gathered}
$$

(28) implies (P-a.s.)

$$
A_{0}(t) \Pi(t, \xi)+A_{0}(t) m_{t}^{(2)}+A_{1}(t) m_{t}=0 .
$$

Consequently, (27),(28) and (29) are the relations from which optimal codings $\left(A_{0}^{*}, A_{1}^{*}\right)$ and the transformator $\Pi^{*}$ are obtained. This completes the proof of the theorem.

Remark. As can be seen from relation (17), the optimal transformator of the back message $\Pi_{0}^{*}$ is constructed in such a way that the back message is multiplied by the value of some deterministic function of time at the moment $t$ and $m_{t}^{(2)}$ with a negative sign, i.e., the noise $\eta_{t}$ is compensated by the best (in the sense of the square criterion) estimate $m_{t}^{(2)}=E\left[\eta_{t} \mid \mathcal{F}_{t}^{\xi}\right]$ (see optimal transmission scheme (18)). 
To conclude this section we can show that condition (15) is indeed a power-type condition.

It can be easily seen that

$$
\begin{gathered}
E\left(A_{0}(t) \widetilde{\xi}_{t}+A_{1}(t) \theta_{t}\right)^{2}=E\left[A_{0}(t) \Pi(t, \xi)+A_{0}(t) m_{t}^{(2)}+A_{1}(t) m_{t}\right]^{2}+ \\
+A_{0}^{2}(t) \gamma_{t}^{(2)}+2 A_{0}(t) A_{1}(t) \gamma_{t}^{(1,2)}+A_{1}^{2}(t) \gamma_{t}=E\left[A_{0}(t) \Pi(t, \xi)+A_{0}(t) m_{t}^{(2)}+\right. \\
\left.+A_{1}(t) m_{t}\right]^{2}+A_{0}^{2}(t) \widetilde{\gamma}_{t}+\left(A_{0}^{2}(t) \gamma_{t}^{(1,2)}+A_{1}(t) \gamma_{t}\right)^{2} \gamma_{t}^{-1}
\end{gathered}
$$

and since $A_{0}^{2}(t) \widetilde{\gamma}_{t} \geq q(t),(30)$ implies

$$
E\left[A_{0}(t) \widetilde{\xi}_{t}+A_{1}(t) \theta_{t}\right]^{2} \geq q(t) .
$$

Consequently (15) implies power condition (31).

\section{$\S 2$. Optimal transmission in a COntinuous case}

Consider the following problem. A Gaussian message $\theta$ is transmitted through an additive white noise instantaneous feedback channel described by the following stochastic differential equation:

$$
d \xi_{t}=A(t, \theta, \widetilde{\xi}) d t+\sigma(t) d w_{t}
$$

where $w=\left(w_{t}, \mathcal{F}_{t}\right)$ is a Wiener process. In contrast to the traditional schemes (see, e.g., [2]-[6]), the feedback here is not assumed to be noiseless.

The functional $A$ in (32) defines the coding, and the back signal $\widetilde{\xi}$ has the following form:

$$
\widetilde{\xi}_{t}=\Pi(t, \xi)+\eta_{t}
$$

where $\Pi$ is the transformator of the back signal, $\eta$ is a noise in the back channel.

In this section optimal transmission schemes are constructed under certain power restrictions in a linear case when $A(t, \theta, \widetilde{\xi})=A_{0}(t) \widetilde{\xi}_{t}+A_{1}(t) \theta_{t}$ and it is proved that these particular linear schemes are also optimal in the general class given by (32),(33).

1. Let the transmitted message $\theta=\left(\theta_{t}, \mathcal{F}_{t}\right), t \in[0, T]$, be a Gaussian process described by the stochastic differential equation

$$
d \theta_{t}=a(t) \theta_{t} d t+b(t) d v_{t}
$$

where $v=\left(v_{t}, \mathcal{F}_{t}\right)$ is a Wiener process independent of the Gaussian $N(m, \gamma)$, $\gamma>0$, random variable $\theta_{0}$, and $|a(t)| \leq k,|b(t)| \leq k$, where $k$ is some constant.

Suppose that $\theta$ is transmitted according to the following linear scheme:

$$
d \xi_{t}=\left[A_{0}(t) \widetilde{\xi}_{t}+A_{1}(t) \theta_{t}\right] d t+\sigma(t) d w_{t}, \quad \xi_{0}=0,
$$


where $w=\left(w_{t}, \mathcal{F}_{t}\right)$ is a Wiener process independent of $v ; A_{0}(t)$ and $A_{1}(t)$ are the coding functions, $\sigma(t)>0$. The back signal $\widetilde{\xi}$ has the form (33). The noise in the back channel $\eta$ admits the stochastic differential

$$
d \eta_{t}=\bar{a}(t) \eta_{t} d t+\bar{b}(t) d \bar{w}_{t}
$$

where $\bar{w}=\left(\bar{w}_{t}, \mathcal{F}_{t}\right)$ is a Wiener process independent of $w$ and $v$ and of the Gaussian $N\left(m_{2}, \gamma_{2}\right), \gamma_{2}>0$, random variable $\eta_{0},|\bar{a}(t)| \leq k,|\bar{b}(t)| \leq k$.

A class of admissible codings, transformator, and decodings is formed by such $\left[\left(A_{0}, A_{1}\right), \Pi, \hat{\theta}\right]$ for which the following conditions are satisfied:

1) the stochastic differential equation (35) has a unique strong solution, $\sup _{t \in[0, T]}\left|A_{i}(t)\right|<\infty, i=0,1$;

2) $E\left[A_{0}(t) \widetilde{\xi}_{t}+A_{1}(t) \theta_{t}\right]^{2} \leq p(t)$, $A_{0}^{2}(t) \widetilde{\gamma}_{t} \geq q(t)$

where $p(t)$ and $q(t)$ are some functions integrable on $[0, T]$ and, for every $t$, $q(t) \leq p(t)$.

Let

$$
\Delta(t)=\inf E\left[\theta_{t}-\hat{\theta}_{t}(\xi)\right]^{2},
$$

where inf is taken in the class of admissible $\left[\left(A_{0}, A_{1}\right), \Pi, \hat{\theta}\right]$.

Theorem 3. When a Gaussian random process $\theta_{t}$ governed by a stochastic differential equation (34) is transmitted through a noisy feedback channel (35),(33),(36) under conditions 1) and 2) optimal in the sense of the square criterion (39), the coding functions $A_{0}^{*}, A_{1}^{*}$ and the transformator of the back message $\Pi^{*}$ have the following form ${ }^{2}$

$$
\begin{gathered}
A_{0}^{*}(t)=\left(\frac{q(t)}{\widetilde{\gamma}_{t}^{*}}\right)^{\frac{1}{2}}, \quad A_{1}^{*}(t)=-\left(\frac{q(t)}{\widetilde{\gamma}_{t}^{*}}\right)^{\frac{1}{2}} \hat{\gamma}_{t}+\left(\frac{p(t)-q(t)}{\gamma_{t}^{*}}\right)^{\frac{1}{2}}, \\
\Pi^{*}\left(t, \xi^{*}\right)=-m_{t}^{*(2)}+\frac{A_{0}^{*}(t)}{A_{1}^{*}(t)} m_{t}^{*},
\end{gathered}
$$

where $\gamma_{t}^{*}$ is equal to the minimal message reproduction error

$$
\begin{gathered}
\Delta(t)=\gamma_{t}^{*}=\gamma \exp \left\{2 \int_{0}^{t} a(s) d s-\int_{0}^{t} \frac{(p(s)-q(s))}{\sigma^{2}(s)} d s\right\}+ \\
+\int_{0}^{t} b(s) \exp \left\{2 \int_{s}^{t} a(u) d s-\int_{s}^{t} \frac{(p(u)-q(u))}{\sigma^{2}(u)} d u\right\} d s,
\end{gathered}
$$

and $\widetilde{\gamma}_{t}^{*}$ and $\hat{\gamma}_{t}^{*}$ are defined by the following equations:

$$
\frac{d \widetilde{\gamma}_{t}^{*}}{d t}=\bar{b}^{2}(t)+2 \widetilde{\gamma}_{t}^{*}\left(\bar{a}(t)-\frac{q(t)}{\sigma^{2}(t)}\right)+\hat{\gamma}_{t}^{*}\left(b^{2}(t)+2 a(t) \gamma_{t}^{*}\right), \quad \widetilde{\gamma}_{0}^{*}=\gamma_{2},
$$

\footnotetext{
${ }^{2}$ The notation of $\S 1$ is used.
} 


$$
\frac{d \hat{\gamma}_{t}^{*}}{d t}=\hat{\gamma}_{t}^{*}\left[\bar{a}(t)-\frac{q(t)}{\sigma^{2}(t)}-b^{2}(t)-2 a(t) \gamma_{t}^{*}\right]-A_{1}^{*}(t) A_{0}^{*}(t) \frac{\widetilde{\gamma}_{t}}{\sigma^{2}(t)}, \quad \hat{\gamma}_{0}^{*}=0 .
$$

The optimal decoding $m_{t}^{*}$ satisfies the following stochastic differential equation:

$$
d m_{t}^{*}=a(t) m_{t}^{*} d t+\left[\gamma_{t}^{*}(p(t)-q(t))\right]^{\frac{1}{2}} \sigma^{-2}(t) d \xi_{t}^{*}, \quad m_{t}^{*}=m .
$$

The optimal transmission is

$$
d \xi_{t}^{*}=\left\{\left(\frac{q(t)}{\widetilde{\gamma}_{t}^{*}}\right)^{\frac{1}{2}} \widetilde{\xi}_{t}^{*}+\left[-\left(\frac{q(t)}{\widetilde{\gamma}_{t}^{*}}\right)^{\frac{1}{2}} \hat{\gamma}_{t}+\left(\frac{p(t)-q(t)}{\gamma_{t}^{*}}\right)^{\frac{1}{2}}\right] \theta_{t}\right\}+\sigma(t) d w_{t}
$$

or

$$
\begin{gathered}
d \xi_{t}^{*}=\left\{\left(\frac{q(t)}{\widetilde{\gamma}_{t}^{*}}\right)^{\frac{1}{2}}\left(\eta_{t}-m_{t}^{*(2)}\right)+\left[-\left(\frac{q(t)}{\widetilde{\gamma}_{t}^{*}}\right)^{\frac{1}{2}} \hat{\gamma}_{t}^{*}+\right.\right. \\
\left.\left.+\left(\frac{p(t)-q(t)}{\gamma_{t}^{*}}\right)^{\frac{1}{2}}\right]\left(\theta_{t}-m_{t}^{*}\right)\right\} d t+\sigma(t) d w_{t}, \quad \xi_{0}^{*}=0,
\end{gathered}
$$

where $m_{t}^{*(2)}$ is defined by the equation

$$
\begin{gathered}
d m_{t}^{*(2)}=\bar{a}(t) m_{t}^{*(2)} d t+\left\{\hat{\gamma}_{t}^{*}\left[\gamma_{t}^{*}(p(t)-q(t))\right]^{\frac{1}{2}}+\right. \\
+\left[q(t) \widetilde{\gamma}_{t}^{*}\right]^{\frac{1}{2}} \sigma^{-2}(t) d \xi_{t}^{*}, \quad m_{0}^{*(2)}=m_{2} .
\end{gathered}
$$

The proof of the theorem is similar to that of an analogous theorem (Theorem 2, §1) for the discrete case, and the equations of nonlinear filtering of conditionally Gaussian type processes (see [2]) are used.

Corollary. When a Gaussian $N(m, \gamma), \gamma>0$, random variable $\theta$ is transmitted through the channel (35),(33),(36), the minimal message reproduction error is

$$
\Delta(t)=\gamma \exp \left[-\int_{0}^{t} \frac{(p(s)-q(s)) d s}{\sigma^{2}(s)}\right] .
$$

Now we shall consider the simplest case of a Gaussian $N(m, \gamma)$ random variable $\theta$ transmission through a noisy feedback channel (32),(33) with $A(t, \theta, \widetilde{\xi})=\widetilde{\xi}_{t}+A(t) \theta$. Let $\sigma(t) \equiv 1$.

For simplicity we assume that $\eta_{t}=\bar{b} w(t)$, where $\bar{b}$ is some constant. Then the optimization problem is simplified and instead of obtaining optimal $A_{0}, A_{1}, \Pi, \hat{\theta}$ as in Part 1 of Section 1 we must find optimal $A, \Pi, \hat{\theta}$.

The necessity of condition (38) is eliminated but it should be required that $p(t) \geq \widetilde{\gamma}_{t}, \quad t \in[0, T]$.

The optimal $A^{*}, \Pi^{*}$, and $\hat{\theta}^{*}$ will have the following form:

$$
A^{*}(t)=-\hat{\gamma}_{t}^{*}+\left(\frac{p(t)-\widetilde{\gamma}_{t}}{\gamma_{t}^{*}}\right)^{\frac{1}{2}},
$$




$$
\Pi^{*}\left(t, \xi^{*}\right)=-m_{t}^{*(2)}+\left[\hat{\gamma}_{t}^{*}-\left(\frac{p-\widetilde{\gamma}_{t}}{\gamma_{t}^{*}}\right)^{\frac{1}{2}}\right] m_{t}^{*}, \quad \hat{\theta}_{t}^{*}=\hat{\theta}_{t}^{*}\left(\xi^{*}\right)=m_{t}^{*},
$$

where $m_{t}^{*}$ admits the representation

$$
d m_{t}^{*}=\left[\gamma_{t}^{*}\left(p(t)-\widetilde{\gamma}_{t}\right)\right]^{\frac{1}{2}} d \xi_{t}^{*}, \quad m_{0}^{*}=m
$$

and $\widetilde{\gamma}_{t}$ and $\hat{\gamma}_{t}$ are found from the relations

$$
\frac{d \widetilde{\gamma}_{t}}{d t}=\bar{b}^{2}-\widetilde{\gamma}_{t}^{2}, \quad \widetilde{\gamma}_{0}=0, \quad \hat{\gamma}_{t}=-\int_{0}^{t}\left(\frac{p(s)-\widetilde{\gamma}_{s}}{\gamma_{s}}\right)^{\frac{1}{2}} \widetilde{\gamma}_{s} d s,
$$

while $m_{t}^{*(2)}$ is found from the stochastic differential equation

$$
d m_{t}^{*(2)}=\left\{\hat{\gamma}_{t}^{*}\left[\gamma_{t}^{*}\left(p(t)-\widetilde{\gamma}_{t}\right)\right]^{\frac{1}{2}}+\widetilde{\gamma}_{t}\right\} d \xi_{t}^{*}, \quad m_{0}^{*(2)}=0 .
$$

The minimal message reproduction error is

$$
\Delta(t)=\gamma \exp \left[-\int_{0}^{t}\left(p(s)-\widetilde{\gamma}_{s}\right) d s\right]
$$

In the case $\bar{b}=1$ we have

$$
\begin{gathered}
\widetilde{\gamma}_{t}=\frac{e^{2 t}-1}{e^{2 t}+1} \\
\Delta(t)=\gamma \exp \left[-\int_{0}^{t} p(s) d s\right] \operatorname{ch} t,
\end{gathered}
$$

where ch $t$ is the hyperbolic cosine.

In the case $\bar{b}=0$, i.e., when the noise $\eta$ in the back channel is absent, we have

$$
\Delta(t)=\gamma \exp \left(-\int_{0}^{t} p(s) d s\right),
$$

which coincides with the transmission through a feedback noiseless channel (see [2]) and our optimal $\Pi$ and $A$ coincide with the optimal codings $A_{0}$ and $A_{1}$ by using the notation of [2], i.e., in this case the transformator $\Pi$ can be placed in the coding device by virtue of a noiseless feedback.

2. Consider the transmission of the Gaussian process described by the stochastic differential equation (34) through the channel (32),(33). Let

$$
\begin{gathered}
\widetilde{A}\left(t, \theta_{t}, \xi\right)=E\left[A\left(t, \theta_{t}, \widetilde{\xi}\right) \mid \mathcal{F}_{t}^{\theta_{t}, \xi}\right], \quad \mathcal{F}^{\theta_{t}, \xi}=\sigma\left\{\theta_{t}, \xi_{s}, s \leq t\right\}, \\
\bar{A}(t, \xi)=E\left[A\left(t, \theta_{t}, \widetilde{\xi}\right) \mid \mathcal{F}_{t}^{\xi}\right] .
\end{gathered}
$$

Assume that the following conditions are satisfied:

1) Equation (1) has a unique strong solution,

2) $E A^{2}\left(t, \theta_{t}, \widetilde{\xi}\right) \leq p(t)$

$E\left[A\left(t, \theta_{t}, \widetilde{\xi}\right)-\widetilde{A}\left(t, \theta_{t}, \xi\right)\right]^{2} \geq q(t)$ 
where $p(t)$ and $q(t)$ are some functions integrable on $[0, T]$ and for every $t$ $p(t) \geq q(t)$.

Let $I_{T}(\theta, \xi)$ be the mutual information of signals $\theta$ and $\xi$ and let $I_{T}(\widetilde{\theta}, \xi)$ be the mutual information of $\widetilde{\theta}=\left(\theta_{t}, \eta_{t}\right)$ and $\xi, t \in[0, T]$.

Lemma 1. The mutual informations $I_{T}(\theta, \xi)$ and $I_{T}(\widetilde{\theta}, \xi)$ have the following forms (see [7]):

$$
\begin{gathered}
I_{T}(\theta, \xi)=\frac{1}{2} \int_{0}^{T} E\left[\widetilde{A}^{2}\left(t, \theta_{t}, \xi\right)-\bar{A}^{2}(t, \xi)\right] \sigma^{-2}(t) d t \\
I_{T}(\widetilde{\theta}, \xi)-I_{T}(\theta, \xi)=\frac{1}{2} \int_{0}^{T} E\left[A\left(t, \theta_{t}, \widetilde{\xi}\right)-\widetilde{A}\left(t, \theta_{t}, \xi\right)\right]^{2} \sigma^{-2}(t) d t .
\end{gathered}
$$

Corollary. Under conditions 1) and 2) we have

$$
I_{T}(\widetilde{\theta}, \xi) \leq \frac{1}{2} \int_{0}^{T} \frac{p(t)}{\sigma^{2}(t)} d t, \quad I_{T}(\widetilde{\theta}, \xi)-I_{T}(\theta, \xi) \geq \frac{1}{2} \int_{0}^{T} \frac{q(t)}{\sigma^{2}(t)} d t .
$$

Let $I_{t}=\sup I_{t}(\theta, \xi)$, where sup is taken in the class of all admissible transmission schemes (32),(33), i.e. schemes for which conditions 1) and 2) are satisfied. Then from the corollary of the lemma we have

$$
I_{t} \leq \frac{1}{2} \int_{0}^{t} \frac{p(s)-q(s)}{\sigma^{2}(s)} d s .
$$

For the linear case $A\left(t, \theta_{t}, \widetilde{\xi}\right)=A_{0}(t) \widetilde{\xi}_{t}+A_{1}(t) \theta_{t}$, since $\bar{A}(t, \xi)=0$ we have

$$
I_{t}\left(\theta, \xi^{*}\right)=\frac{1}{2} \int_{0}^{t} E \widetilde{A}^{2}\left(s, \theta_{s}, \xi^{*}\right) d s=\frac{1}{2} \int_{0}^{t} \frac{p(s)-q(s)}{\sigma^{2}(s)} d s .
$$

Hence the following theorem is true.

Theorem 4. The optimal codings $A_{0}^{*}, A_{1}^{*}$, the decoding $m_{t}^{*}$ and the transformator $\Pi^{*}$ constructed in Theorem 3 are also optimal in the sense of maximum of mutual information.

3. Finally we prove the following

Theorem 5. When a Gaussian $N(m, \gamma), \gamma>0$, random variable $\theta$ is transmitted according to the transmission scheme (32),(33) under conditions 1) and 2), the minimal reproduction error $\delta(t)=\inf _{(A, \Pi, \hat{\theta})} E\left(\theta-\hat{\theta}_{t}(\xi)\right)^{2}$ has the following form:

$$
\delta(t)=\Delta(t)=\gamma \exp \left\{-\int_{0}^{t}(p(s)-q(s)) \sigma^{-2} d s\right\},
$$


where $\Delta(t)$ is the minimal message reproduction error for the optimal linear transformation constructed in Theorem 3 , i.e. among all admissible schemes the transmission constructed in Theorem 3 is optimal in the sense of the square criterion.

Proof. Since $\delta(t) \leq \Delta(t)$, the theorem will be proved if we show that

$$
\delta(t) \geq \gamma \exp \left\{-\int_{0}^{t}(p(s)-q(s)) \sigma^{-2} d s\right\} .
$$

Let $\hat{\theta}=\hat{\theta}_{t}(\xi)$ be some decoding. Then by Lemma 16.8 from [2] we have

$$
E\left(\theta-\hat{\theta}_{t}(\xi)\right)^{2} \geq \gamma e^{-2 I\left(\theta, \hat{\theta}_{t}(\xi)\right)} .
$$

But $I\left(\theta, \hat{\theta}_{t}(\xi)\right) \leq I_{t}(\theta, \xi)$, and according to Theorem 4

$$
I_{t}(\theta, \xi) \leq I_{t}\left(\theta, \xi^{*}\right)=\frac{1}{2} \int_{0}^{t}(p(s)-q(s)) \sigma^{-2}(s) d s .
$$

Hence inequality (43) holds and Theorem 5 is true.

\section{REFERENCES}

1. O.A.Glonti, Investigations in the theory of conditionally Gaussian processes.(Russian) Metsniereba, Tbilisi, 1985.

2. R.Sh.Liptser and A.N.Shiryayev, Statistics of random processes: I. General Theory, II. Applications. Springer, Berlin, 1977, 1978 (transl. from Russian, Nauka, Moscow, 1974).

3. S.Ihara, Coding theory in white Gaussian channel with feedback. $J$. Multivariate Anal. 4(1974), 74-87.

4. S.Ihara, Coding theory in Gaussian channel with feedback. II. Evaluation of the filtering error. Nagoya Math. J. 58(1975), 127-147.

5. P.K.Katyshev, Transmission of Gaussian process over a feedback channel. (Russian) Problemi Peredachi Informatsii 12(1976), No. 1, 112-118.

6. R.S.Liptser, Optimal coding and decoding in transmission of Gaussian Markov message through channel with noiseless feedback. (Russian) Problemi Peredachi Informatsii 10(1974), No. 4, 3-15.

7. O.A.Glonti, On mutual information of signals in the case of transmission over a "noisy" feedback channel. (Russian) Teor. Veroyatnost. $i$ primenen. 23(1978), No. 2, 395-397; English translation: Theory Probab. Appl. 23(1978), 377-379.

Author's address:

(Received 25.12.1992)

Laboratory of Probabilistic and Statistical Methods, I.Javakhishvili Tbilisi State University,

150a David Agmashenebeli Ave., Tbilisi 380012

Republic of Georgia 